\title{
Terbium glows green
}

\author{
Geng Deng relates how terbium, a garden-variety lanthanide, has found its way into our daily lives owing \\ to its green phosphorescence.
}

\begin{abstract}
$t$ may be one of the rarer rare-earth elements in the Earth's crust, but terbium is actually quite common around us. Despite their name, the rare-earth elements (which consist of the lanthanides as well as scandium and yttrium) aren't all that scarce; element 65 for example is more abundant than mercury, and as numerous lights and monitors of modern life have made use of green terbium-based phosphors, it has seeped into our homes and working environments.
\end{abstract}

The story of terbium discovery started in Ytterby, a prolific small village in Sweden that has no fewer than four rare-earth elements named directly after it - yttrium, terbium, erbium and ytterbium - and from whose earths (as oxides were then called) several other elements were also isolated (scandium, thulium, holmium, gadolinium and lutetium). It took decades to identify the composition of a black mineral found in Ytterby by Carl Axel Arrhenius in 1787, first referred to as ytterbite. It was later renamed gadolinite after Johan Gadolin, who realized it contained an unknown earth; that is, the oxide of a new element, which he called yttria.

In 1843 Swedish chemist Carl Gustaf Mosander separated an yttria sample into three components: yttria (mostly consisting of yttrium oxide), as well as erbia and terbia, each of which, he believed, contained a new element. He was right — but a mixup of Mosander's samples during their spectroscopic analysis means that terbium was isolated from his original erbia, while erbium was found in his terbia. This was just too confusing, so the minerals later also swapped names to match those of their principal components.

Like the other lanthanides, terbium's most common oxidation state is +III. The green (or light-lime) phosphorescence under ultraviolet light of its trivalent salts, such as $\mathrm{Tb}_{2}\left(\mathrm{SO}_{4}\right)_{3}$ (pictured), has long been known - it is so intense that it can be seen with the naked eye. It arises from several excited-to-

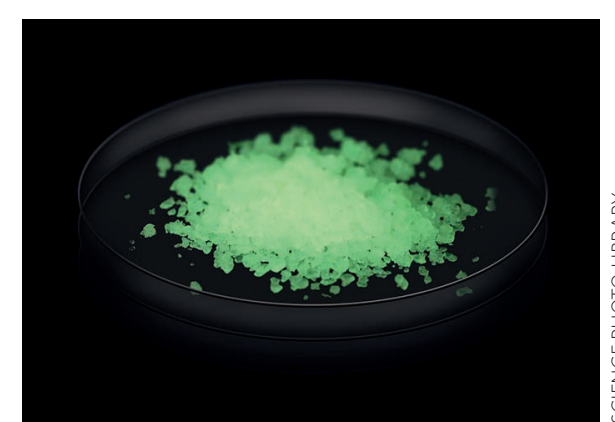

ground electronic-state transitions ${ }^{1}$, with one transition contributing the main emission peak at $545 \mathrm{~nm}$. Many of its rare-earth cousins also luminesce brightly in different colours; for example, europium(III) is a red emitter while its reduced form europium(II) is a blue one. But although those emissions were well-known, it wasn't until the second part of the twentieth century that they were taken advantage of for practical applications in lighting.

The incandescent lamp, invented by Thomas Edison in 1879, had been widely used for over a century. But it is inherently energy-inefficient: heating a wire with an electric current to the point that it will glow means that most of the energy used is dissipated in the form of heat and only a small fraction is converted into light. In the 1960 s, rare-earth salts came into focus ${ }^{2,3}$ as they waste little energy during the emission process. Furthermore, the green-emitting terbium compounds could be combined with the red-and blue-emitting europium ones to make white-light fluorescent lamps. The first commercial such rare-earth-based energyefficient lamp was made in 1974, and this type of lighting soon spread across the world.

Meanwhile, traditional colour television sets and monitors were also being developed that used cathode ray tubes, in which electron beams are sent to the screen to excite phosphors and in turn generate images. Here as well the combination of terbium and europium compounds in the red-green-blue (RGB) additive colour model allowed a wide range of colours to be produced. Between the 1950s and 1990s, those monitors delivered terbium compounds to millions of households around the globe. Although the advent of flat-panel technologies in the late 2000s has curbed that application, terbiumcontaining compounds have also found use as probes in the biomedical field, such as in fluoroimmunoassays ${ }^{4}$ and supramolecular luminescent sensors ${ }^{5}$.

A perhaps more exotic use of element 65 is in an alloy called terfenol-D, which consists of terbium, iron and dysprosium: it is a magnetostrictive material that contracts and expands in a magnetic field. Because it is able to withstand high strains, terfenol-D has been used in actuators and underwater transducers. It also serves in the portable system 'SoundBug', which can be attached to any resonant flat surface (such as, wood, glass or metal) to turn it into a speaker: the magnetostrictive material converts electrical input into vibrations by changing the applied magnetic field, which are then amplified by the resonant surface.

Located in the middle of the lanthanides - a part of the periodic table that has remained relatively unexplored - terbium has shone through owing to its distinctive green phosphorescence and found exciting uses.

GENG DENG is a graduate student in the Department of Chemistry, Tsinghua University, Beijing 100084, China. He is also one of the authors of the textbook: Physical Organic Chemistry: Structure and Principle. e-mail:dengg13@mails.tsinghua.edu.cn.

\section{References}

1. Andres, J. \& Chauvin A.-S. in The Rare Earth Elements (ed. Atwood, D. A.) $135-152$ (John Wiley \& Sons, 2012)

2. Ropp, R. C. J. Electrochem. Soc. 111, 311-317 (1964)

3. Wanmaker, W. L. \& Bril, A. Philips Res. Repts. 19, 479-497 (1964).

4. Moore, E. G. Samuel, A. P. S. \& Raymond K. N. Acc. Chem. Res. 42, 542-552 (2009)

5. dos Santos, C. M. G. Harte, A. J. Quinn, S. J. \& Gunnlaugsson T. Coord. Chem. Rev. 252, 2512-2527 (2008).

Corrected after print: 31 January 2018 


\section{Correction}

In the In Your Element 'Terbium glows green' (Nat. Chem. 10, 110; 2018), phosphorescence was mistakenly described as fluorescence in three instances: the standfirst, the fourth paragraph, and the final paragraph. Furthermore, the line from the fourth paragraph "Many of its rare-earth cousins also fluoresce" was changed to "Many of its

rare-earth cousins also luminesce". This has been corrected after print 31 January 2018. 